\title{
Numerical modelling of wave energy converters
}

\author{
Verbrugghe Tim, Brecht Devolder, PeterTroch and Andreas Kortenhaus \\ Coastal Engineering, Bridges and Roads; Civil Engineering Department; Ghent University, \\ Technologiepark 904, 9052 Zwijnaarde, Belgium \\ E-mail: timl.verbrugghe@ugent.be
}

\section{Introduction}

In the last century, research into renewable energy resources became more and more important. Where wind and solar energy are already commercially viable, wave energy is a rather new, but promising development. The power within ocean waves is dense, can efficiently travel long distances and is sufficient to contribute significantly to the energy market. Wave energy from ocean waves is absorbed by using Wave Energy Converters (WECs). WEC development is characterised by a large variety of device types; differentiation is made based on the location, device geometry, mooring type and operating method. In this research, focus is put on wave activated bodies. These are floating devices, located nearshore or offshore, producing energy directly from their own kinetic and potential energy, in response to the incoming waves. In order to extract a considerable amount of wave power at a location in a cost-effective way, large numbers of WECs are arranged in farms using a particular geometrical configuration. Interactions between the individual WECs (near field effects) affect the overall power production of the farm. In response to an incoming wave, a floating body starts moving in six degrees of freedom. As a consequence, the device itself starts creating waves, called radiated waves. Additionally, because of the presence of a structure in the wave field, the incoming waves are partially reflected and diffracted. The total wave field around a WEC is thus a combination of incoming, radiated, reflected and diffracted waves. This results into zones with higher wave elevations and zones with lower wave elevations compared to the incident wave field. One should thus avoid that one WEC is positioned in the wake region of another WEC. By arranging the WEC park layout with the individual WECs positioned in zones with amplified wave elevations, the total power production can be significantly higher. In combination with these near field effects, the installation of a WEC park also influences the wave climate further away (far field effects). The wave height reduction behind an entire WEC farm affects other users in the sea, the environment or even the coastline. When installed close enough to the shoreline, a WEC farm can be used as a first-line coastal defence mechanism.

\section{Numerical modelling}

In order to answer these fundamental underlying questions on WEC farm design, we aim to develop a methodology and a numerical tool, by using coupled numerical models, supported by experimental validation data. Applying these models could lead to finding the optimal and cost-effective configurations of WEC farms for power production, and quantifying the related environmental impact. The novelty of this approach is that a numerical model, suited for near field effects will be developed and validated. Several modelling types are considered. Firstly, CFD modelling with OpenFOAM is applied, which takes turbulence, viscosity and non-linearity into account, but has a high computational cost. Alternatively, a linear/non-linear potential flow solver (BEM Method) can be used, which neglects turbulence and viscosity, but runs fast and efficient. Furthermore, it will be combined with a numerical wave propagation model suited for predicting far field effects for two purposes: to reduce the computational cost of the near field model and to get more accurate results of the far field effect of WECs. Several models can be applied for propagating the waves in the far field: the mild-slope model MILDwave, the non-hydrostatic model SWASH, or the fully non-linear potential flow model OceanWave3D. The resulting coupled numerical tool will allow us to draw reliable conclusions regarding optimal and cost-effective design of farms of wave energy converters.

Keywords: waves; wave energy; numerical modelling; coupled models 